\title{
Smaller pineal gland is associated with rapid eye movement sleep behavior disorder in Alzheimer's disease
}

Jeongbin Park ${ }^{1 \dagger}$, Seung Wan Suh ${ }^{2 \dagger}$, Grace Eun Kim', Subin Lee ${ }^{1}$, Jun Sung Kim', Hye Sung Kim³, Seonjeong Byun ${ }^{3}$, Jong Bin Bae ${ }^{3}$, Jae Hyoung Kim ${ }^{4,5}$, Sang Eun Kim ${ }^{6,7}$, Ji Won $\mathrm{Han}^{3}$ and Ki Woong Kim,3,8*

\begin{abstract}
Background: To investigate the association between pineal gland volume and symptoms of rapid eye movement (REM) sleep behavior disorder (RBD) in Alzheimer's disease (AD) patients without any feature of dementia with Lewy bodies.

Methods: We enrolled 296 community-dwelling probable AD patients who did not meet the diagnostic criteria for possible or probable dementia with Lewy bodies. Among them, 93 were amyloid beta (Aß) positive on ${ }^{18} \mathrm{~F}-$ florbetaben amyloid brain positron emission tomography. We measured RBD symptoms using the REM Sleep Behavior Disorder Screening Questionnaire (RBDSQ) and defined probable RBD (pRBD) as the RBDSQ of 5 or higher. We manually segmented pineal gland on 3T structural T1-weighted brain magnetic resonance imaging.

Results: The participants with pRBD had smaller pineal parenchyma volume (VPP) than those without pRBD $(p<0.001)$. The smaller the VPP, the more severe the RBD symptoms $(p<0.001)$. VPP was inversely associated with risk of prevalent pRBD (odds ratio $=0.909,95 \%$ confidence interval $[\mathrm{Cl}]=0.878-0.942, p<0.001$ ). Area under the receiver operator characteristic curve for pRBD of VPP was 0.80 (95\% Cl=0.750-0.844, $p<0.0001$ ). These results were not changed when we analyzed the 93 participants with $A \beta$-positive $A D$ separately.
\end{abstract}

Conclusions: In AD patients, reduced pineal gland volume may be associated with RBD.

Keywords: Alzheimer's disease, Pineal gland, Rapid eye movement sleep behavior disorder, Magnetic resonance imaging, Amyloid positron emission tomography

\section{Background}

Rapid eye movement (REM) sleep behavior disorder (RBD) is a parasomnia characterized by loss of normal skeletal muscle atonia accompanied by dream-enacting behaviors [1]. A large autopsy study found that $94 \%$ of

\footnotetext{
* Correspondence: kwkimmd@snu.ac.kr

${ }^{\dagger}$ Jeongbin Park and Seung Wan Suh contributed equally to this work.

${ }^{1}$ Department of Brain and Cognitive Sciences, Seoul National University

College of Natural Sciences, Seoul, Korea

${ }^{3}$ Department of Neuropsychiatry, Seoul National University Bundang Hospital, Seongnam, Korea

Full list of author information is available at the end of the article
}

the neurodegenerative disorders associated with RBD were synucleinopathies and claimed that the presence of RBD should at least raise suspicion of primary or coexisting Lewy body disease even in the typical Alzheimer's disease (AD) [2]. However, their claim seems somewhat overextended. First of all, their study sample may not represent overall RBD and be subject to a sampling bias [2]. Second, RBD is also prevalent in cognitively normal older adults [3], suggesting that RBD may occur without synucleinopathy. AD is far more prevalent than synucleinopathies [4], and RBD was quite common in numerous cross-sectional and prospective studies on AD patients

(c) The Author(s). 2020 Open Access This article is licensed under a Creative Commons Attribution 4.0 International License, which permits use, sharing, adaptation, distribution and reproduction in any medium or format, as long as you give appropriate credit to the original author(s) and the source, provide a link to the Creative Commons licence, and indicate if changes were made. The images or other third party material in this article are included in the article's Creative Commons licence, unless indicated otherwise in a credit line to the material. If material is not included in the article's Creative Commons licence and your intended use is not permitted by statutory regulation or exceeds the permitted use, you will need to obtain permission directly from the copyright holder. To view a copy of this licence, visit http://creativecommons.org/licenses/by/4.0/. The Creative Commons Public Domain Dedication waiver (http://creativecommons.org/publicdomain/zero/1.0/) applies to the data made available in this article, unless otherwise stated in a credit line to the data. 
[5-15]. There is no reason to assume that AD patients will develop RBD only from synucleinopathy, not the pathologies that can lead to RBD in normal older adults without synucleinopathy.

In cognitively normal older adults, the smaller pineal gland was associated with more RBD symptoms and higher risk of incident RBD symptoms, suggesting that reduction of melatonin secretion associated with the reduction of pineal gland volume may be a potential cause of RBD [16]. AD patients show reduced endogenous melatonin levels [17] and have a smaller pineal gland compared to healthy controls [18]. Pineal gland is a small neuroendocrine organ, and its primary function is to regulate sleep through the synthesis and secretion of melatonin [19]. In humans, roughly $80 \%$ of the pineal gland comprises melatonin-producing pinealocytes [19], and pineal gland volume is proportional to the endogenous melatonin levels [20,21]. Pineal gland volume can be changed by various physiological or pathological conditions that may change melatonin production [16, 22]. In a couple of clinical trials, RBD symptoms such as dream-enacting behaviors and REM sleep muscle atonia were improved by the administration of melatonin [23, 24] but relapsed by its discontinuation [23]. However, the association between pineal gland and RBD has never been investigated in AD patients.

In this study, we investigated the association between pineal gland volume and RBD symptoms in probable AD patients who did not meet the diagnostic criteria of possible and probable dementia with Lewy bodies (DLB) [25].

\section{Methods}

\section{Participants}

We enrolled 296 community-dwelling probable AD from the visitors to the Dementia Clinic of the Seoul National University Bundang Hospital (SNUBH) from 2011 to 2020. Among them, 104 underwent a ${ }^{18} \mathrm{~F}$-florbetaben amyloid brain positron emission tomography (PET) scan, and 93 were found to be amyloid beta $(\mathrm{A} \beta)$-positive.

We excluded the participants with following conditions: possible or probable DLB or Parkinson's disease dementia (PDD); any major psychiatric and/or neurological disorders that could affect cognitive function other than AD; any history of brain tumors, substance abuse or dependence, and use of medications such as clonazepam or exogenous melatonin that may influence RBD symptom; any serious medical conditions that could affect the structure and/or function of the pineal gland or abnormalities in pineal gland morphology such as neoplastic lesions or extremely large cystic gland (diameter greater than $15.0 \mathrm{~mm}$ ) [26]; and those with high risk of restless legs syndrome (positive on Cambridge-Hopkins Restless Legs Syndrome questionnaire) [27] and obstructive sleep apnea (STOP-BANG questionnaire score of $\geq 5$ points) [28], all of which could mimic symptoms of RBD $[29,30]$.

All participants were fully informed with the protocol of this study, and provided written informed consents signed by themselves or their legal guardians. This study was approved by the Institutional Review Board of the SNUBH.

\section{Diagnostic assessments}

Geriatric psychiatrists with expertise in dementia research conducted in person standardized diagnostic interviews, detailed medical histories, and physical/ neurological examinations using the Korean version of the Consortium to Establish a Registry for Alzheimer's Disease Assessment Packet Clinical Assessment Battery (CERAD-K) [31] and the Korean version of the MiniInternational Neuropsychiatric Interview [32]. Additionally, research neuropsychologists administered the CERA D-K Neuropsychological Assessment Battery (CERADK-N) [31, 33], Digit Span Test [34], Frontal Assessment Battery [35], and Geriatric Depression Scale [36].

Trained research nurses collected data on age, sex, years of education, duration of AD (months), intracranial volume (ICV), history of head injury, amount of smoking (packs/day) and alcohol drinking (standard units/week) over the past 12-month period, and use of drugs influencing sleep or motor activity, including cholinesterase inhibitors (donepezil, rivastigmine, and galantamine), antidepressants (selective serotonin reuptake inhibitor, serotonin norepinephrine reuptake inhibitor, and others), carbamazepine, triazolam, zopiclone, quetiapine, clozapine, and sodium oxybate to each participant. We diagnosed dementia according to the fourth edition of the Diagnostic and Statistical Manual of Mental Disorders Text Revision criteria [37]. Global severity of dementia was determined according to the Clinical Dementia Rating [38]. We determined probable AD according to the National Institute of Neurological and Communicative Disorders and Stroke/Alzheimer's Disease and Related Disorders Association diagnostic criteria [39]. We diagnosed probable or possible DLB and PDD according to the diagnostic criteria proposed by McKeith et al. [25], in which the presence of RBD features was ignored in the current study.

\section{Assessment of brain amyloid deposition}

We performed ${ }^{18} \mathrm{~F}$-florbetaben amyloid brain PET imaging using a Discovery VCT scanner (General Electric Medical Systems; Milwaukee, WI, USA) in threedimensional (3D) acquisition mode. The participants were injected with $8.1 \mathrm{mCi}(300 \mathrm{MBq})$ of ${ }^{18} \mathrm{~F}$-florbetaben (Neuraceq) as a slow single intravenous bolus $(6 \mathrm{~s} / \mathrm{mL})$ in a total volume of up to $10 \mathrm{~mL}$. After a 90-min uptake period, we obtained 20-min PET images comprising four 
5-min dynamic frames. The determination was based on the visual interpretation of tracer uptake in the gray matter of the following four brain regions: the temporal lobes, frontal lobes, posterior cingulate cortex/ precuneus, and parietal lobes. Participants were considered $A \beta$ positive if smaller areas of tracer uptake were equal to or higher than those present in the white matter extending beyond the white matter rim to the outer cortical margin involving the majority of the slices within at least one of the four brain regions ("moderate" $\mathrm{A} \beta$ deposition) or a large confluent area of tracer uptake (i.e., signal intensity) was equal to or higher than that present in the white matter extending beyond the white matter rim to the outer cortical margin and involving the entire region including the majority of slices within at least one of the four brain regions ("pronounced" A $\beta$ deposition). Participants were considered $A \beta$ negative if tracer uptake in the gray matter is lower than that in the white matter in all four brain regions (no $\beta$-amyloid deposition).

\section{Assessment of rapid eye movement sleep behavior disorder symptoms}

We evaluated behavioral features of RBD using the REM Sleep Behavior Disorder Screening Questionnaire (RBDSQ) [40]. The RBDSQ is a self-reported screening instrument used to diagnose RBD and comprises 10 items assessing the most prominent clinical features of RBD: items 1 to 4 , the frequency and content of dreams and their relationship to nocturnal movements and behaviors; item 5 , self-injuries and injuries to the bed partner; item 6, four subsections specifically assessing nocturnal motor behavior (e.g., questions about nocturnal vocalization (6.1), sudden limb movements (6.2), complex movements (6.3), or bedside items that fall down (6.4)); items 7 and 8, nocturnal awakenings; item 9, disturbed sleep in general; and item 10, the presence of any neurological disorder. Each item could be answered as "yes" or "no." The RBDSQ score ranges from 0 to 13 points, with higher scores indicating more features associated with RBD. We defined probable RBD (pRBD) as having a total score of 5 or higher on the RBDSQ [40]. The questionnaire was completed by the participants with the corroboration from their partners.

\section{Assessment of pineal gland volume}

We obtained 3D structural T1-weighted spoiled gradient echo magnetic resonance (MR) images using a Philips 3.0 Tesla Achieva scanner (Philips Medical Systems; Eindhoven, the Netherlands) within 3 months of clinical assessments with the following parameters: acquisition voxel size $=1.0 \times 0.5 \times 0.5 \mathrm{~mm}$; sagittal slice thickness = $1.0 \mathrm{~mm}$; repetition time $=4.61 \mathrm{~ms}$; echo time $=8.15 \mathrm{~ms}$; number of excitations $=1$; flip angle $=8^{\circ}$; field of view $=$
$240 \times 240 \mathrm{~mm}$; and acquisition matrix size $=175 \times 256 \times$ $256 \mathrm{~mm}$ in the $x$-, $y$-, and $z$-dimensions. We implemented bias field correction to remove the signal intensity inhomogeneity artifacts of MR images using Statistical Parametric Mapping software (version 12, SPM12; Wellcome Trust Centre for Neuroimaging, London; http://www.fil. ion.ucl.ac.uk/spm). We resliced the MR images into an isotropic voxel size of $1.0 \times 1.0 \times 1.0 \mathrm{~mm}^{3}$. We measured ICV using FreeSurfer software (version 5.3.0; http://surfer. nmr.mgh.harvard.edu) to adjust for interindividual variabilities in brain volume. We assessed pineal gland volume as described in our previous work [16]. In brief, trained researchers constructed a 3D mask of each pineal gland by manually segmenting the pineal gland slice-by-slice on the resliced T1-weighted MR images at $1.0 \times 1.0 \times 1.0 \mathrm{~mm}^{3}$ using the ITK-SNAP software (version 3.4.0; http://www. itksnap.org). We measured pineal gland volume and pineal cysts volume and estimated the volume of pineal parenchyma (VPP) by subtracting the pineal cysts volume from the pineal gland volume (Fig. 1). We defined a pineal cyst as an area of homogenous intensity that was isointense to the cerebrospinal fluid in T1 sequence images with a diameter of $2.0 \mathrm{~mm}$ or greater [41].

The intra-rater and inter-rater intraclass correlation coefficient were 0.983 (95\% confidence interval $[\mathrm{CI}]=$ 0.956-0.993, $p<0.001)$ and 0.934 $(\mathrm{CI}=0.828-0.974$, $p<0.001)$, respectively.

\section{Statistical analyses}

We compared the continuous variables using the independent samples $t$ tests and categorical variables using the chi-squared tests between groups. We compared VPP between the participants with pRBD and those without $\mathrm{pRBD}$ using analysis of covariance that adjusted for age, sex, years of education, ICV, head injury, smoking, alcohol drinking, and use of drugs influencing sleep or motor activity as covariates. We examined the association between VPP and the risk of pRBD using binary logistic regression analysis that was adjusted for the same covariates. We examined the diagnostic performance of the VPP for pRBD using the receiver operating characteristic (ROC) analysis. We calculated the optimal cutoff value and area under the curve (AUC) using Youden index maximum (sensitivity + specificity -1 ). We examined the association of VPP with RBDSQ total score (RBDSQ- $\mathrm{T}$ ) and the item-6 score of the RBDSQ (RBDSQ-6) using multiple linear regression model adjusted for the covariates stated above. The RBDSQ item 6 comprises four subitems on the core symptoms of RBD: nocturnal vocalization (6.1), sudden limb movements (6.2), complex movements (6.3), or bedside items that fall down (6.4). We conducted the same analyses only on the $\mathrm{A} \beta$-positive $\mathrm{AD}$ patients. 


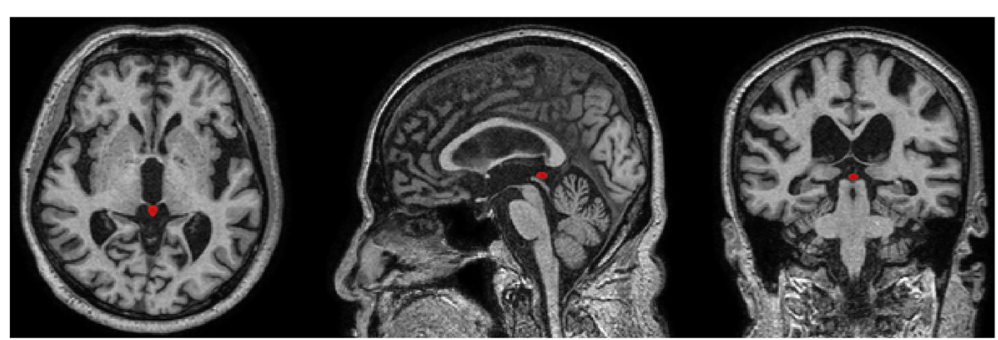

Fig. 1 Assessment of pineal gland volume on 3D T1-weighted brain magnetic resonance images at $1.0 \times 1.0 \times 1.0 \mathrm{~mm}^{3}$. The pineal gland was manually segmented from surrounding cerebrospinal fluid space

For all analyses, we considered a two-tailed $p$ value less than 0.05 as statistically significant and employed Bonferroni corrections to reduce type I error when multiple comparisons were conducted. We performed ROC analyses using MedCalc for Windows version 18.11.3 (MedCalc Software, Mariakerke, Belgium) and all the other statistical analyses using the Statistical Package for the Social Sciences for Windows version 20.0 (International Business Machines Corporation, Armonk, NY).

\section{Results}

As summarized in Table 1, the AD patients with $\mathrm{pRBD}$ showed smaller VPP than those without pRBD $(p<$ 0.001). VPP was inversely associated with the risk of
pRBD (odds ratio $[\mathrm{OR}]=0.909,95 \% \mathrm{CI}=0.878-0.942$, $p<0.001$ ), indicating that the $\mathrm{AD}$ patients with smaller VPP may be more likely to have pRBD than those with smaller VPP. The AUC of VPP for pRBD was 0.80 (95\% $\mathrm{CI}=0.750-0.844, p<0.0001$, Fig. $2 \mathrm{a})$, and the optimal cutoff value for classifying pRBD was $62 \mathrm{~mm}^{3}$ (sensitivity $=87.18 \%$; specificity $=58.75 \%$ ). VPP was also inversely associated with the RBDSQ-T (standardized $\beta=-0.410$, $p<0.001$ ) and the RBDSQ-6 (standardized $\beta=-0.224$, $p<0.001$, Fig. 3a).

These results were not changed when we analyzed the $\mathrm{A} \beta$-positive $\mathrm{AD}$ patients separately. Among the 93 participants with $\mathrm{A} \beta$-positive AD, 11 (11.83\%) had pRBD. The $A \beta$-positive AD patients with pRBD showed smaller

Table 1 Demographic and clinical characteristics of the participants

\begin{tabular}{|c|c|c|c|}
\hline & Without pRBD $(n=257)$ & With pRBD $(n=39)$ & $p$ \\
\hline Age (years, mean $\pm S D$ ) & $77.4 \pm 7.4$ & $76.8 \pm 7.4$ & $0.634^{a}$ \\
\hline Sex (women, \%) & 69.3 & 79.5 & $0.191^{\mathrm{a}}$ \\
\hline Education (years, mean \pm SD) & $9.9 \pm 5.6$ & $8.1 \pm 5.5$ & $0.065^{\mathrm{a}}$ \\
\hline Presence of cohabitants, (present, \%) & 80.5 & 74.4 & $0.371^{\mathrm{a}}$ \\
\hline Duration of $\mathrm{AD}$ (months, mean $\pm \mathrm{SD}$ ) & $36.9 \pm 25.7$ & $44.0 \pm 37.8$ & $0.265^{\mathrm{a}}$ \\
\hline Drugs influencing sleep or motor activity (users, \%) & 29.2 & 38.5 & $0.240^{a}$ \\
\hline History of head injury (present, \%) & 9.0 & 10.3 & $0.792^{\mathrm{a}}$ \\
\hline Alcohol drinking (SU/week, mean \pm SD) & $1.8 \pm 7.1$ & $0.7 \pm 3.4$ & $0.375^{a}$ \\
\hline Smoking (packs/day, mean \pm SD) & $0.1 \pm 0.6$ & $0.0 \pm 0.2$ & $0.750^{\mathrm{a}}$ \\
\hline GDS (points, mean \pm SD) & $12.2 \pm 6.9$ & $16.5 \pm 6.7$ & $<0.001^{\mathrm{a}}$ \\
\hline CDR (points, mean \pm SD) & $0.7 \pm 0.4$ & $0.9 \pm 0.5$ & $0.903^{\mathrm{a}}$ \\
\hline STOP-BANG (points, mean \pm SD) & $2.3 \pm 0.9$ & $2.6 \pm 1.0$ & $0.041^{\mathrm{a}}$ \\
\hline \multicolumn{4}{|l|}{ RBDSQ (points, mean \pm SD) } \\
\hline Total score & $1.4 \pm 1.2$ & $6.1 \pm 1.4$ & $<0.001^{a}$ \\
\hline Item-6 score & $0.2 \pm 0.5$ & $1.2 \pm 1.2$ & $<0.001^{a}$ \\
\hline Intracranial volume $\left(\mathrm{cm}^{3}\right.$, mean $\left.\pm S D\right)$ & $1515.5 \pm 147.7$ & $1509.1 \pm 154.1$ & $0.805^{a}$ \\
\hline $\operatorname{VPP}\left(\mathrm{mm}^{3}\right.$, mean $\left.\pm S D\right)$ & $69.5 \pm 18.5$ & $51.7 \pm 10.8$ & $<0.001^{\mathrm{b}}$ \\
\hline Cerebral amyloid deposition (present, \%) & 31.9 & 28.2 & $0.643^{\mathrm{a}}$ \\
\hline
\end{tabular}

Abbreviations: $P R B D$ probable REM sleep behavior disorder, SD standard deviation, $A D$ Alzheimer's disease, SU standard units, GDS Geriatric Depression Scale, CDR Clinical Dementia Rating, RBDSQ REM Sleep Behavior Disorder Screening Questionnaire, VPP pineal parenchyma volume

andependent sample $t$ test for continuous variables and chi-square test for categorical variables

${ }^{\mathrm{b}}$ Analysis of covariance adjusted for age, sex, years of education, intracranial volume, head injury, amount of smoking, amount of alcohol drinking, and use of drugs influencing sleep or motor activity 

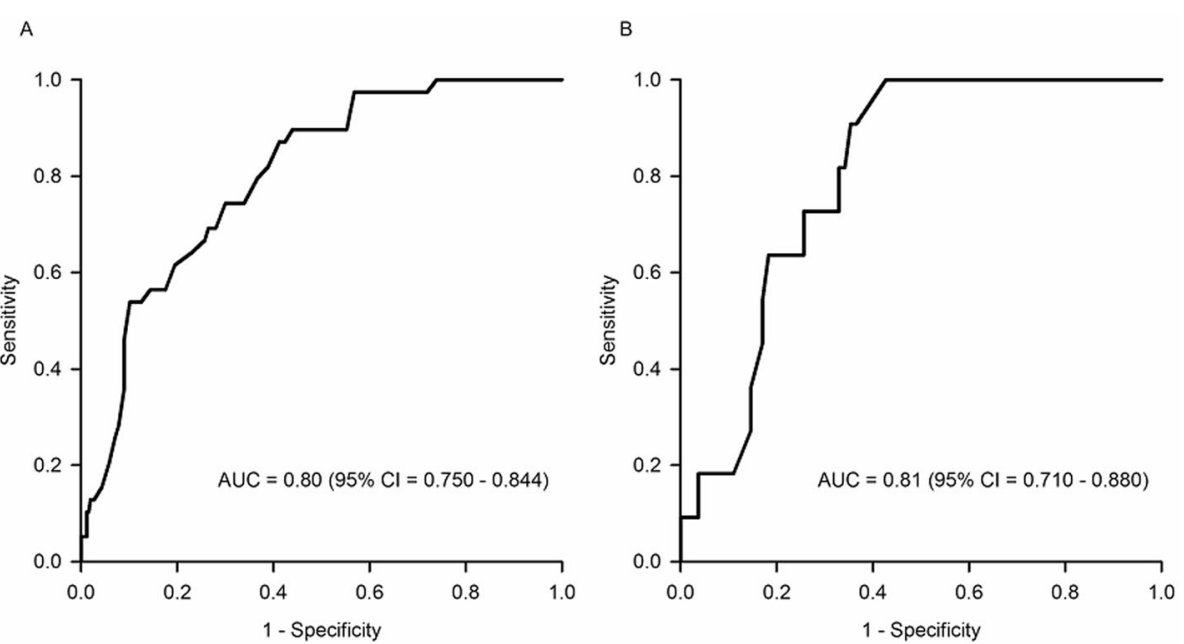

Fig. 2 Diagnostic accuracy for the prevalent probable rapid eye movement sleep behavior disorder of the pineal parenchyma volume in a all participants and $\mathbf{b}$ participants with A $\beta$-positive Alzheimer's disease. A $\beta$, amyloid beta; VPP, pineal parenchyma volume $\left(\mathrm{mm}^{3}\right)$; $A U C$, area under the curve; $\mathrm{Cl}$, confidence interval

VPP than those without pRBD $(p=0.002)$. VPP was inversely associated with the risk of $\mathrm{pRBD}(\mathrm{OR}=0.901,95 \%$ $\mathrm{CI}=0.840-0.966, p=0.004)$, and AUC of VPP for $\mathrm{pRBD}$ was 0.81 (95\% CI $=0.710-0.880, p<0.0001$; Fig. $2 b)$. The optimal cutoff value of the VPP for classifying pRBD was $60 \mathrm{~mm}^{3}$ (sensitivity $=100 \%$; specificity $=57.32 \%$ ). VPP also showed significant inverse association with the RBDSQ-T (standardized $\beta=-0.491, p<0.001$ ) and the RBDSQ-6 (standardized $\beta=-0.276, p=0.015$, Fig. 3b).

\section{Discussion}

In this cross-sectional study, we found that smaller pineal parenchyma volume was associated with more RBD symptoms in AD patients, which is in line with our previous observation that smaller pineal parenchyma volume was associated with the more RBD symptoms and the higher risk of future pRBD in cognitively normal older adults [16].

It is now well established that RBD is a strong predictor of neurodegeneration, in particular $\alpha$ synucleinopathies [1]. According to a previous clinicopathological study, $94 \%$ of the polysomnography (PSG)confirmed RBD patients were found to have synucleinopathies at autopsy [2], suggesting that the presence of RBD in patients with dementia may favor the diagnosis of DLB [42]. However, not all RBD patients progressed

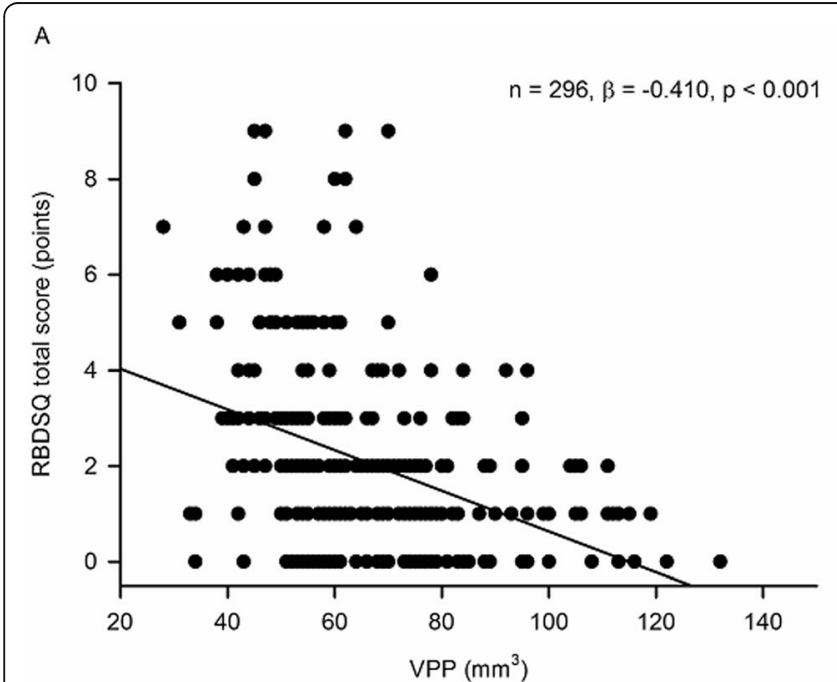

B

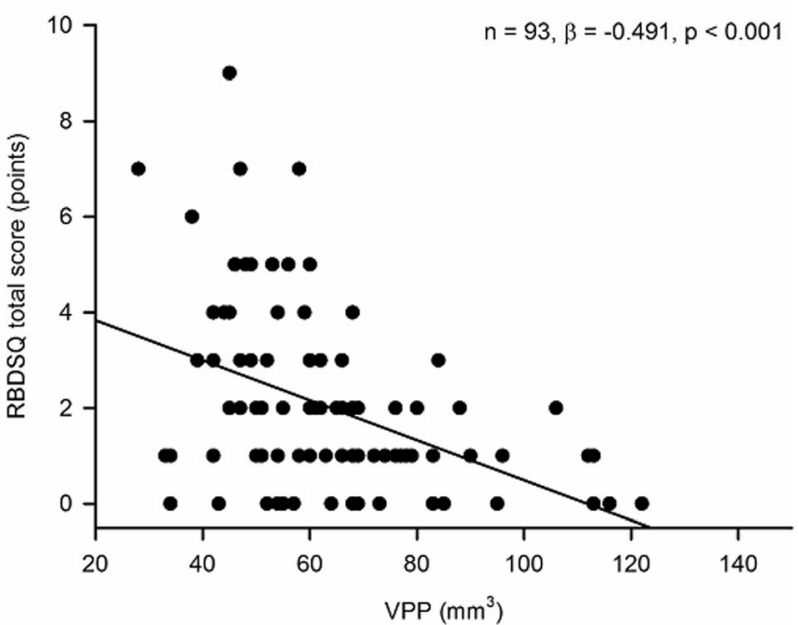

Fig. 3 Association between REM Sleep Behavior Disorder Screening Questionnaire total score and pineal parenchyma volume $\left(\mathrm{mm}^{3}\right)$ in $\mathbf{a}$ all participants and $\mathbf{b}$ participants with A $\beta$-positive Alzheimer's disease. Multiple linear regression model adjusted for age, sex, years of education, intracranial volume, head injury, amount of smoking, amount of alcohol drinking, and use of drugs influencing sleep or motor activity 
to neurodegenerative syndrome with synucleinopathies. The overall conversion rate from idiopathic RBD to an overt neurodegenerative syndrome was $6.3 \%$ per year in the elderly adults aged $66.3 \pm 8.4$ years on average [43] Furthermore, RBD can occur alone without any neurological conditions, and large clinical series have reported that the idiopathic form of RBD accounts for up to $60 \%$ of the cases [3]. Therefore, we should be more cautious in confirming that all dementia with RBD is a synucleinopathy or at least a neurodegenerative disease having synucleinopathies as a secondary pathology. Although synucleinopathies may be a common sufficient condition for RBD, it is not a necessary condition for RBD.

RBD was common in clinically diagnosed $\operatorname{AD}[5,6]$ and $3-11 \%$ of polysomnography-defined RBD patients developed AD [9-14]. In amyloid PET-confirmed AD patients, 24.6\% showed RBD in a previous study [8], and $11.8 \%$ showed pRBD in the current study. Some authors have argued that an imbalance of acetylcholine transmission, a hallmark of $\mathrm{AD}$, could explain the occurrence of $\mathrm{RBD}$ in a small portion of AD patients [6]. This is based on the findings that acetylcholine may be involved in the induction of REM sleep atonia [15], considering that an injection of cholinergic agonists induced muscle atonia in dogs [44] and the administration of cholinesterase inhibitors augmented the amount of REM sleep [45]. The brainstem regions also have been implicated in RBD pathophysiology based on lesion studies in animals, especially involving pontine nuclei including the noradrenergic locus coeruleus (LC), cholinergic pedunculopontine nucleus, and laterodorsal tegmental nucleus [1]. Lesioning the LC causes REM sleep without atonia, and size of the lesion determines whether simple or complex behaviors are exhibited [46]. The LC is prone to early neurodegeneration [47], and LC neurons can be lost up to $70 \%$ in AD brains [48]. Therefore, atrophy of LC nuclei with impaired noradrenergic systems may also contribute to the development of $\mathrm{RBD}$ in $\mathrm{AD}$ patients [6].

In our previous and the current works, we demonstrated the association of smaller pineal gland with the risk of pRBD in both cognitively normal older adults without any symptom or sign of neurodegenerative disorders including synucleinopathies [16] and in AD patients without any symptom or sign of synucleinopathies. These results suggested that reduced endogenous melatonin production may be another cause of RBD in $\mathrm{AD}$ patients as well as in normal older adults because the secretion of melatonin was strongly associated with pineal gland volume. Compared to healthy controls, $\mathrm{AD}$ patients showed disrupted circadian melatonin rhythm, lower melatonin levels in the cerebrospinal fluid, serum and postmortem pineal gland [17], and smaller pineal parenchyma [18]. Since the pineal gland is a circumventricular organ surrounded by the cerebrospinal fluid [19], it can be easily influenced by soluble $\mathrm{A} \beta$ peptides [49]. A previous in vitro study of isolated rat pineal glands confirmed that $A \beta$ directly inhibited pineal melatonin synthesis and impaired melatonergic systems, leading to a neuroinflammatory response within the gland [49]. Therefore, enduring insults of $A \beta$ may reduce pineal gland volume and melatonin production, which may increase the risk of RBD in AD patients. In addition, under physiological conditions, melatonin in vivo protects central cholinergic neurons against $A \beta$ mediated toxicity via its antioxidant and antiamyloidogenic properties [50]. Melatonin not only inhibits $A \beta$ generation but also arrests the formation of amyloid fibrils by a structure-dependent interaction with A $\beta$ [50]. Therefore, reduced melatonin production due to pineal atrophy may also increase the risk of RBD or worsen RBD symptoms in AD patients indirectly via reduced protection of the cholinergic system from amyloid toxicity.

\section{Limitations}

Our study has several methodological limitations. First, we used a questionnaire to determine if a participant was at a high risk of RBD, whereas video PSG is required to establish the definitive diagnosis of RBD [1]. This could be a substantial problem when the participants have significant cognitive impairments such as AD, leading to a recall bias. However, considering that the previous reports have suggested that the prevalence of PSGconfirmed $\mathrm{RBD}$ in $\mathrm{AD}$ subjects ranges from $5 \%$ (mean age [SD], 70.5 [9.4]; mean disease duration of $\mathrm{AD}, 16.1$ [7.1] months) [6] to $27 \%$ (mean age, 70.2 [5.6] with global deterioration scale score of 3 or 4 [5], our results, with the prevalence of pRBD of $13 \%$ (mean age, 77.3 [7.4]; mean disease duration of AD, 37.8 [27.6] months), seem to be in a reasonable extent. Additionally, we obtained the RBDSQ data with the corroboration from the participant's partners, which could increase their validity. Second, although we strictly excluded AD patients who simultaneously met the diagnostic criteria for possible or probable DLB, it is still possible that our study samples could have included the patients with synucleinopathies because clinical features between AD and DLB are overlapping [51] and $40-50 \%$ of $\mathrm{AD}$ patients had $\alpha$ synuclein-positive Lewy bodies [52-54]. In addition, we did not conduct brain dopamine transporter scan or metaiodobenzylguanidine myocardial scan which would have helped to rule out DLB more definitively. However, even in synucleinopathies, the pineal gland may be associated with the risk of RBD because melatonin also played a protective role against synucleinopathies [55]. Third, causal relationship between pineal gland volume 
and pRBD cannot be inferred because the current study employed a cross-sectional design.

\section{Conclusion}

In conclusion, the current study suggests that smaller pineal gland may be associated with the risk and/or severity of $R B D$ in $A D$ patients.

\section{Abbreviations}

AD: Alzheimer's disease; AUC: Area under the curve; $A \beta$ : Amyloid beta; CERA D: Consortium to Establish a Registry for Alzheimer's Disease; Cl: Confidence interval; DLB: Dementia with Lewy bodies; ICC: Intraclass correlation coefficient; ICV: Intracranial volume; LC: Locus coeruleus; MRI: Magnetic resonance imaging; OR: Odds ratio; PDD: Parkinson's disease dementia; PET: Positron emission tomography; pRBD: Probable rapid eye movement sleep behavior disorder; PSG: Polysomnography; RBD: Rapid eye movement sleep behavior disorder; RBDSQ: Rapid Eye Movement Sleep Behavior Disorder Screening Questionnaire; RBDSQ-6: Item-6 score of the RBDSQ RBDSQ-T: RBDSQ total score; REM: Rapid eye movement; ROC: Receiver operating characteristic; SD: Standard deviation; SNUBH: Seoul National University Bundang Hospital; SU: Standard units; VPP: Volume of pineal parenchyma

\section{Acknowledgements}

Not applicable

\section{Authors' contributions}

All authors contributed to the study concept and design. JP, SWS, and KWK analyzed the data. JP, SWS, and KWK drafted the manuscript. All authors contributed to the interpretation of the data, review of the drafts of the manuscript, and approval of the final version.

\section{Funding}

This study was supported by the grants from the Korean Health Technology R\&D Project, Ministry of Health and Welfare, Republic of Korea (grant no. HI09C1379 [A092077]) and the Institute for Information \& Communications Technology Promotion (IITP) grant funded by the Korea government (MSIT) (2018-2-00861, Intelligent SW Technology Development for Medical Data Analysis).

\section{Availability of data and materials}

The datasets used/or analyzed during the current study are available from the corresponding author on reasonable request.

\section{Ethics approval and consent to participate}

All participants were fully informed with the protocol of this study and provided written informed consents signed by themselves or their legal guardians. This study was approved by the Institutional Review Board of the Seoul National University Bundang Hospital.

\section{Consent for publication}

Not applicable

\section{Competing interests}

The authors declare that they have no competing interests.

\section{Author details}

${ }^{1}$ Department of Brain and Cognitive Sciences, Seoul National University College of Natural Sciences, Seoul, Korea. ${ }^{2}$ Department of Psychiatry, Kangdong Sacred Heart Hospital, Hallym University College of Medicine, Seoul, Korea. ${ }^{3}$ Department of Neuropsychiatry, Seoul National University Bundang Hospital, Seongnam, Korea. ${ }^{4}$ Department of Radiology, Seoul National University Bundang Hospital, Seongnam, Korea. ${ }^{5}$ Department of Radiology, Seoul National University College of Medicine, Seoul, Korea. ${ }^{6}$ Department of Nuclear Medicine, Seoul National University Bundang Hospital, Seongnam, Korea. ${ }^{7}$ Department of Nuclear Medicine, Seoul National University College of Medicine, Seoul, Korea. ${ }^{8}$ Department of Psychiatry, Seoul National University College of Medicine, Seoul, Korea.
Received: 7 August 2020 Accepted: 11 November 2020

Published online: 21 November 2020

\section{References}

1. Boeve BF. REM sleep behavior disorder: updated review of the core features, the REM sleep behavior disorder-neurodegenerative disease association, evolving concepts, controversies, and future directions. Ann N Y Acad Sci. 2010;1184(1):15-54.

2. Boeve BF, Silber M, Ferman TJ, Lin S, Benarroch E, Schmeichel A, et al. Clinicopathologic correlations in 172 cases of rapid eye movement sleep behavior disorder with or without a coexisting neurologic disorder. Sleep Med. 2013;14(8):754-62.

3. Fantini ML, Ferini-Strambi L, Montplaisir J. Idiopathic REM sleep behavior disorder: toward a better nosologic definition. Neurology. 2005;64(5):780-6.

4. Kim KW, Park JH, Kim MH, Kim MD, Kim BJ, Kim SK, et al. A nationwide survey on the prevalence of dementia and mild cognitive impairment in South Korea. J Alzheimers Dis. 2011;23(2):281-91.

5. Gagnon J-F, Petit D, Fantini ML, Rompré S, Gauthier S, Panisset M, et al. REM sleep behavior disorder and REM sleep without atonia in probable Alzheimer disease. Sleep. 2006:29(10):1321-5.

6. Wang P, Wing YK, Xing J, Liu Y, Zhou B, Zhang Z, et al. Rapid eye movement sleep behavior disorder in patients with probable Alzheimer's disease. Aging Clin Exp Res. 2016;28(5):951-7.

7. Kim H-J, Im HK, Kim J, Han J-Y, De Leon M, Deshpande A, et al. Brain atrophy of secondary REM-sleep behavior disorder in neurodegenerative disease. J Alzheimers Dis. 2016:52(3):1101-9.

8. Kim H-S, Lee HJ, Shin D-J, Lee Y-B, Noh Y, Park KH. The prevalence of rapid eye movement sleep behavior disorder in amyloid positron emission tomography positive Alzheimer's disease. J Sleep Med. 2019;16(2):102-8.

9. Schenck $\mathrm{CH}$, Boeve BF, Mahowald MW. Delayed emergence of a parkinsonian disorder or dementia in $81 \%$ of older men initially diagnosed with idiopathic rapid eye movement sleep behavior disorder: a 16-year update on a previously reported series. Sleep Med. 2013;14(8):744-8.

10. Youn S, Kim T, Yoon I-Y, Jeong J, Kim HY, Han JW, et al. Progression of cognitive impairments in idiopathic REM sleep behaviour disorder. J Neurol Neurosurg Psychiatry. 2016;87(8):890-6.

11. Wing YK, Li SX, Mok V, Lam SP, Tsoh J, Chan A, et al. Prospective outcome of rapid eye movement sleep behaviour disorder: psychiatric disorders as a potential early marker of Parkinson's disease. J Neurol Neurosurg Psychiatry. 2012;83(4):470-2.

12. Zhou J, Zhang J, Lam SP, Chan JW, Mok V, Chan A, et al. Excessive daytime sleepiness predicts neurodegeneration in idiopathic REM sleep behavior disorder. Sleep. 2017;40(5):Zsx041

13. Postuma R, Gagnon J, Vendette M, Fantini M, Massicotte-Marquez J, Montplaisir J. Quantifying the risk of neurodegenerative disease in idiopathic REM sleep behavior disorder. Neurology. 2009;72(15):1296-300.

14. Postuma R, Gagnon J-F, Rompré S, Montplaisir J. Severity of REM atonia loss in idiopathic REM sleep behavior disorder predicts Parkinson disease. Neurology. 2010;74(3):239-44.

15. Galbiati A, Carli G, Hensley M, Ferini-Strambi L. REM sleep behavior disorder and Alzheimer's disease: definitely no relationship? J Alzheimers Dis. 2018; 63(1):1-11.

16. Park J, Han JW, Suh SW, Byun S, Han JH, Bae JB, et al. Pineal gland volume is associated with prevalent and incident isolated rapid eye movement sleep behavior disorder. Aging (Albany N Y). 2020;12(1):884-93.

17. Wu YH, Swaab DF. The human pineal gland and melatonin in aging and Alzheimer's disease. J Pineal Res. 2005;38(3):145-52.

18. Matsuoka T, Imai A, Fujimoto H, Kato Y, Shibata K, Nakamura K, et al. Reduced pineal volume in Alzheimer disease: a retrospective cross-sectional MR imaging study. Radiology. 2017;286(1):239-48.

19. Reiter RJ. The mammalian pineal gland: structure and function. Am J Anat. 1981;162(4):287-313.

20. Liebrich LS, Schredl M, Findeisen P, Groden C, Bumb JM, Nölte IS Morphology and function: MR pineal volume and melatonin level in human saliva are correlated. J Magn Reson Imaging. 2014;40(4):966-71.

21. Nölte I, Lütkhoff AT, Stuck BA, Lemmer B, Schredl M, Findeisen $P$, et al. Pineal volume and circadian melatonin profile in healthy volunteers: an interdisciplinary approach. J Magn Reson Imaging. 2009;30(3):499-505.

22. Park J, Han JW, Lee JR, Byun S, Suh SW, Kim T, et al. Lifetime coffee consumption, pineal gland volume, and sleep quality in late life. Sleep. 2018;41(10):Zsy127. 
23. Kunz D, Bes F. Melatonin as a therapy in REM sleep behavior disorder patients: an open-labeled pilot study on the possible influence of melatonin on REM-sleep regulation. Mov Disord. 1999;14(3):507-11.

24. Boeve BF, Silber MH, Ferman TJ. Melatonin for treatment of REM sleep behavior disorder in neurologic disorders: results in 14 patients. Sleep Med. 2003:4(4):281-4

25. McKeith IG, Dickson D, Lowe J, Emre M, O'brien J, Feldman H, et al. Diagnosis and management of dementia with Lewy bodies third report of the DLB consortium. Neurology. 2005;65(12):1863-72.

26. Osborn AG, Preece MT. Intracranial cysts: radiologic-pathologic correlation and imaging approach. Radiology. 2006;239(3):650-64.

27. Allen RP, Burchell BJ, MacDonald B, Hening WA, Earley CJ. Validation of the self-completed Cambridge-Hopkins questionnaire ( $\mathrm{CH}-\mathrm{RLSq})$ for ascertainment of restless legs syndrome (RLS) in a population survey. Sleep Med. 2009;10(10):1097-100

28. Chung F, Elsaid $\mathrm{H}$. Screening for obstructive sleep apnea before surgery: why is it important? Curr Opin Anaesthesiol. 2009;22(3):405-11.

29. Gaig C, Iranzo A, Pujol M, Perez H, Santamaria J. Periodic limb movements during sleep mimicking REM sleep behavior disorder: a new form of periodic limb movement disorder. Sleep. 2017;40(3):zsw063.

30. Iranzo A, Santamaría J. Severe obstructive sleep apnea/hypopnea mimicking REM sleep behavior disorder. Sleep. 2005;28(2):203-6.

31. Lee JH, Lee KU, Lee DY, Kim KW, Jhoo JH, Kim JH, et al. Development of the Korean Version of the Consortium to Establish a Registry for Alzheimer's Disease Assessment Packet (CERAD-K) clinical and neuropsychological assessment batteries. J Gerontol Ser B Psychol Sci Soc Sci. 2002;57(1):47-53.

32. Yoo S-W, Kim Y-S, Noh J-S, Oh K-S, Kim C-H, NamKoong K, et al. Validity of Korean version of the mini-international neuropsychiatric interview. Anxiety Mood. 2006;2:50-5.

33. Lee DY, Lee KU, Lee JH, Kim KW, Jhoo JH, Kim SY, et al. A normative study of the CERAD neuropsychological assessment battery in the Korean elderly. J Int Neuropsychol Soc. 2004;10(1):72-81.

34. Wechsler D. Instruction Manual for the Wechsler Memory Scale Revised. New York: Psychological Corporation; 1987.

35. Kim TH, Huh Y, Choe JY, Jeong JW, Park JH, Lee SB, et al. Korean version of frontal assessment battery: psychometric properties and normative data. Dement Geriatr Cogn Disord. 2010;29(4):363-70.

36. Kim JY, Park JH, Lee JJ, Huh Y, Lee SB, Han SK, et al. Standardization of the Korean version of the geriatric depression scale: reliability, validity, and factor structure. Psychiatry Investig. 2008;5(4):232-8.

37. American Psychiatric Association. Diagnostic and statistical manual of mental disorders, 4th edition, text revision. Washington, DC: American Psychiatric Association Press; 2000.

38. Hughes CP, Berg L, Danziger W, Coben LA, Martin RL. A new clinical scale for the staging of dementia. Br J Psychiatry. 1982;140(6):566-72

39. McKhann G, Drachman D, Folstein M, Katzman R, Price D, Stadlan EM. Clinical diagnosis of Alzheimer's disease report of the NINCDS-ADRDA Work Group* under the auspices of Department of Health and Human Services Task Force on Alzheimer's Disease. Neurology. 1984;34(7):939.

40. Stiasny-Kolster K, Mayer G, Schäfer S, Möller JC, Heinzel-Gutenbrunner M, Oertel WH. The REM sleep behavior disorder screening questionnaire-a new diagnostic instrument. Mov Disord. 2007;22(16):2386-93.

41. Pu Y, Mahankali S, Hou J, Li J, Lancaster J, Gao J-H, et al. High prevalence of pineal cysts in healthy adults demonstrated by high-resolution, noncontrast brain MR imaging. AJNR Am J Neuroradiol. 2007;28(9):1706-9.

42. Ferman TJ, Boeve BF, Smith G, Silber M, Kokmen E, Petersen RC, et al. REM sleep behavior disorder and dementia: cognitive differences when compared with AD. Neurology. 1999;52(5):951.

43. Postuma RB, Iranzo A, Hu M, Hogl B, Boeve BF, Manni R, et al. Risk and predictors of dementia and parkinsonism in idiopathic REM sleep behaviour disorder: a multicentre study. Brain. 2019;142(3):744-59.

44. Nishino S, Tafti M, Reid MS, Shelton J, Siegel JM, Dement WC, et al. Muscle atonia is triggered by cholinergic stimulation of the basal forebrain: implication for the pathophysiology of canine narcolepsy. J Neurosci. 1995; 15(7):4806-14

45. Mizuno S, Kameda A, Inagaki T, Horiguchi J. Effects of donepezil on Alzheimer's disease: the relationship between cognitive function and rapid eye movement sleep. Psychiatry Clin Neurosci. 2004;58(6):660-5.

46. Hendricks JC, Morrison AR, Mann GL. Different behaviors during paradoxical sleep without atonia depend on pontine lesion site. Brain Res. 1982;239(1): 81-105.
47. Mravec B, Lejavova K, Cubinkova V. Locus (coeruleus) minoris resistentiae in pathogenesis of Alzheimer's disease. Curr Alzheimer Res. 2014;11(10):9921001.

48. Bondareff W, Mountjoy CQ, Roth M. Loss of neurons of origin of the adrenergic projection to cerebral cortex (nucleus locus ceruleus) in senile dementia. Neurology. 1982;32(2):164.

49. Cecon E, Chen M, Marçola M, Fernandes PA, Jockers R, Markus RP. Amyloid $\beta$ peptide directly impairs pineal gland melatonin synthesis and melatonin receptor signaling through the ERK pathway. FASEB J. 2015;29(6):2566-82.

50. Rosales-Corral SA, Acuña-Castroviejo D, Coto-Montes A, Boga JA, Manchester LC, Fuentes-Broto L, et al. Alzheimer's disease: pathological mechanisms and the beneficial role of melatonin. J Pineal Res. 2012;52(2): 167-202.

51. Walker Z, Jaros E, Walker RW, Lee L, Costa DC, Livingston G, et al. Dementia with Lewy bodies: a comparison of clinical diagnosis, FP-CIT single photon emission computed tomography imaging and autopsy. J Neurol Neurosurg Psychiatry. 2007;78(11):1176-81.

52. Hamilton RL. Lewy bodies in Alzheimer's disease: a neuropathological review of 145 cases using a-synuclein immunohistochemistry. Brain Pathol. 2000;10(3):378-84.

53. Arai $Y$, Yamazaki M, Mori O, Muramatsu H, Asano G, Katayama Y. aSynuclein-positive structures in cases with sporadic Alzheimer's disease: morphology and its relationship to tau aggregation. Brain Res. 2001;888(2): 287-96.

54. Lippa CF, Schmidt ML, Lee VMY, Trojanowski JQ. Antibodies to a-synuclein detect Lewy bodies in many Down's syndrome brains with Alzheimer's disease. Ann Neurol. 1999;45(3):353-7.

55. Brito-Armas JM, Baekelandt V, Castro-Hernandez JR, Gonzalez-Hernandez T, Rodriguez M, Castro R. Melatonin prevents dopaminergic cell loss induced by lentiviral vectors expressing A30P mutant alpha-synuclein. Histol Histopathol. 2013;28(8):999-1006.

\section{Publisher's Note}

Springer Nature remains neutral with regard to jurisdictional claims in published maps and institutional affiliations.
Ready to submit your research? Choose BMC and benefit from:

- fast, convenient online submission

- thorough peer review by experienced researchers in your field

- rapid publication on acceptance

- support for research data, including large and complex data types

- gold Open Access which fosters wider collaboration and increased citations

- maximum visibility for your research: over $100 \mathrm{M}$ website views per year

At BMC, research is always in progress.

Learn more biomedcentral.com/submissions 